\title{
Design and Implementation of FMCW Surveillance Radar Based on Dual Chirps
}

\author{
Youngseok Jin, Bongseok Kim, Sangdong Kim, Jonghun Lee \\ ART Lab., Daegu Gyeongbuk Institute of Science \& Technologies, \\ Techno-Jungangdaero 333, Daegu, South Korea \\ jhlee@dgist.ac.kr
}

\begin{abstract}
We propose and implement a low-complexity dual-chirp FMCW radar system for surveillance applications. The FMCW radar is suitable for the detection of various positions of multiple targets for the monitoring of concealed humans. For a surveillance FMCW radar system, it is necessary to distinguish between stationary and moving targets while maintaining a low level of complexity. However, conventional FMCW radar systems are very complex with many chirps to distinguish between stationary and moving targets. Even in hardware with low complexity levels, in order to distinguish between a stationary and a moving target, the proposed algorithm employs only dual chirps. Experimental results show that the proposed algorithm can effectively distinguish between moving and stationary targets despite its low complexity and low-level hardware.
\end{abstract}

Index Terms-FMCW; Radar; Surveillance; Low complexity.

\section{INTRODUCTION}

Recently, there have been several studies of radar sensors for surveillance applications [1]-[10]. These areas are popular because radar sensors have many advantages compared to other sensors. For example, radar sensors can detect at longer distances than an ultrasonic sensor with a short detection distance and can detect targets even in environments in which a camera sensor cannot operate easily, such as at night or in fog [1]-[3].

Meanwhile, in the various radar systems used for surveillance applications, the frequency-modulated continuous wave (FMCW) radar system represents a promising technology due to advantages such as lower complexity and cost levels [6]-[15]. Compared to pulse radar systems, FMCW radar systems have relatively low transmitted peak power levels and can meet certain range and velocity resolution requirements with relatively simple hardware and architectures [6].

In earlier work [10]-[12], FMCW radar algorithms with multiple channels for surveillance were designed and implemented. In one study [10], the authors investigated the implementation of $24 \mathrm{GHz}$ FMCW radar for surveillance and detected the range, velocity and angle of targets. Meanwhile, in other words [11]-[13], researchers presented a scalable

Manuscript received 14 December, 2017; accepted 10 August, 2018.

This work was supported in DGIST was supported in part by the DGIST R\&D Program of the Ministry of Science, ICT and Future Planning, Korea (18-IT-01). architecture for acquisition and a FPGA-based processing platform of a radar sensor with a single transmitter and multiple receivers. However, these algorithms usually perform full-dimensional FFT when detecting in order to distinguish between stationary and moving targets and thus require high complexity levels. The most important issue in relation to surveillance radar is a quick identification of the presence of a moving target. Therefore, these algorithms are not suitable for surveillance radar systems due to their high complexity.

In this paper, we propose and implement a low-cost FMCW radar algorithm with very low complexity for surveillance applications. The proposed radar algorithm recognizes a moving target with an inexpensive chip and a simple RF. In order to distinguish between stationary and moving targets, we initially employ only dual chirps. Due to the characteristics of FMCW radar signals, when the target moves, the phase according to the chirp changes due to the Doppler effect. That is, the proposed algorithm recognizes the existence of the target by observing the phase change between the dual chirp signals. Furthermore, this system is implemented as a $24 \mathrm{GHz}$ module with low complexity in order to verify that the proposed algorithm is suitable for surveillance applications.

The structure of the paper is as follows. In Section II, we introduce and define the system model and the main notations used in order to ensure a proper understanding of the FMCW algorithm, such as the TX and RX signals used in FMCW radar systems. Section III shows the conventional FMCW radar detection algorithm using the 3D FFT. In Section IV, we present the structure of the proposed algorithm. In Sections V and VI, simulation and experimental results for various cases are provided. Finally, Section VII concludes this paper.

\section{SySTEM MODELS}

This section discusses the different models of FMCW radar systems and the notations used in them. We consider reflected signals of FMCW radar in order to detect the ranges, the velocities, and the azimuth distributions of targets [6]. To facilitate an easier understanding of the concepts, we initially consider the case of a single target, after which we extend this to the case of multiple targets.

Consider a uniform linear array (ULA) of $K$ receiving (RX) antenna arrays with distance $d$ between adjacent arrays 
and a single transmitted (TX) antenna. The TX FMCW signal denoted by $x_{0}(t)$ is shown in Fig. 1(a) and is expressed as

$$
x_{0}(t)=\exp \left(j 2 \pi\left(f_{0} t+\mu t^{2} / 2\right)\right)
$$

where $0 \leq t \leq T, f_{0}$ is the carrier frequency, $T$ is the frequency modulation period during which the TX frequency sweeps over the entire bandwidth $B$, and $\mu$ is the chirp rate, i.e., $\mu=B / T$. All $L$ chirp signals are transmitted during a frame; i.e., $T_{F}=T L$. The signal reflected from the target is received with delay $\tau$ and direction of arrival (DOA) $\theta$ at the $k$-th array, as shown in Fig. 2. Here, the $l$-th chirp slot is denoted by $x_{l, k}(t)$ and is expressed as

$$
\begin{gathered}
x_{l, k}(t)=a_{0} x_{0}(t-\tau) \exp \left(j 2 \pi f_{D} l T\right) \times \\
\times \exp (j 2 \pi d k \sin (\theta) / \lambda)+\tilde{w}_{l, k}(t),
\end{gathered}
$$

where $a_{0}$ is the complex amplitude, $\tilde{w}_{l, k}$ is the additive white Gaussian noise (AWGN) signal, $f_{D}$ is the Doppler frequency due to the moving of the target and $\lambda$ is the wavelength. By denoting the velocity term as $v^{l}\left(f_{D}\right)=\exp \left(j 2 \pi l f_{D} T\right)$ and the DOA term as $\psi^{k}(\theta)=\exp (j 2 \pi d k \sin (\theta) / \lambda), x_{l, k}(t)$ can simply be represented as follows

$$
x_{l, k}(t)=a_{0} x_{0}(t-\tau) v^{l}\left(f_{D}\right) \psi^{k}(\theta)+\tilde{w}_{l, k}(t) .
$$



(a) TX signal (dashed line) and RX signal (solid line)

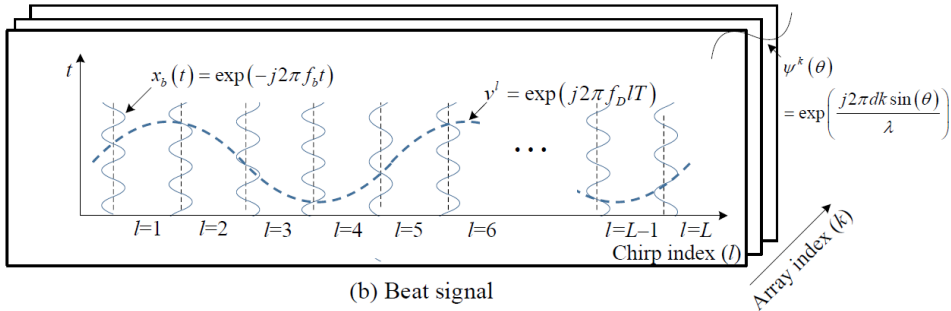

Fig. 1. Waveforms of FMCW radar.

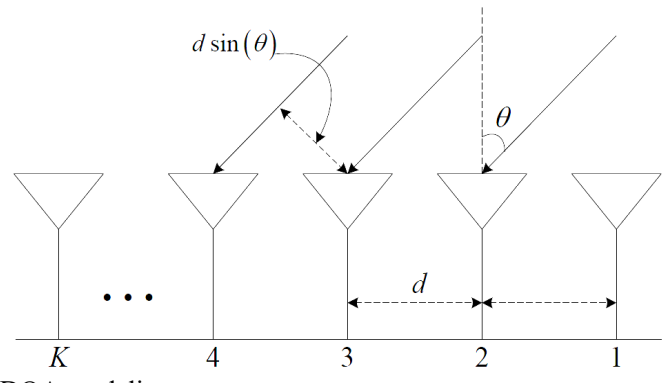

Fig. 2. DOA modeling.

The beat signal $y_{l, k}(t)$ is expressed by omitting $\theta$ and $f_{D}$ for simplicity, as follows

$$
\begin{gathered}
y_{l, k}(t)=x_{l, k}(t) \times x_{0}^{*}(t)= \\
=a \exp \left(-j 2 \pi f_{b} t\right) v^{l} \psi^{k}+w_{l, k}(t)= \\
=a x_{b}(t) v^{l} \psi^{k}+w_{l, k}(t),
\end{gathered}
$$

where $f_{b}=\mu \tau$ is the beat frequency and $x_{b}(t)$ is the sinusoid term in the beat signal. In addition, $a$ and $w_{l, k}(t)$ are expressed as follows:

$$
\begin{gathered}
a=a_{0} \exp \left(-j 2 \pi\left(f_{0} \tau-\mu \tau^{2} / 2\right)\right), \\
w_{l, k}(t)=\tilde{w}_{l, k}(t) x_{0}^{*}(t) .
\end{gathered}
$$

After an analogue-to-digital conversion (ADC) of $y_{l, k}(t)$, the discrete time model of (4) with the sampling frequency $f_{s}=1 / T_{s}$ is denoted by $y_{l, k}[n]$; i.e., $y_{l, k}[n]=y_{l, k}\left(n T_{s}\right)$ for $n=0,1, \ldots, N_{s}-1$ where $t_{s}$ is the sampling time interval and $N_{s}=T / T_{s}$ and $y_{l, k}[n]$ can be represented as follows

$$
y_{l, k}[n]=a x_{b}\left[n T_{s}\right] v^{l} \psi^{k}+w_{l, k}[n]
$$

\section{CONVEntional 3D -FFT Detection Algorithm FoR SURVEILLANCE APPLICATIONS}

This section introduces the conventional detection algorithm using the 3D-FFT. In order to estimate the range, the velocity and the angle of a target, a data cube composed of the 3D beat signal in (7) is employed. The conventional algorithm performs 3D-FFT operations on the data cube in the time (range) domain, the ramp (chirp) domain, and the array domain.

First, as shown in Fig. 3, a $N_{\text {RFFT-point FFT operation on }}$ the data cube in the time (range) domain is performed, where $N_{\text {RFFT }}$ denotes the number of points of the FFT in the range domain

$$
Y_{l, k}[u]=\sum_{n=0}^{N_{\mathrm{s}}-1} y_{l, k}[n] W_{N_{\mathrm{RFFT}}}^{q n},
$$

where $0 \leq u \leq N_{\text {RFFT }}-1, \quad W_{N}$ is the $N$-point DFT 
operation; i.e., $W=\exp (-j 2 \pi / N)$. That is, the range domain $N_{\text {RFFT-point }}$ FFT output vector at the $l$-th chirp and the $k$-th array $\mathbf{Y}_{l, k}=\left[Y_{l, k}[1], Y_{l, k}[2], \ldots, Y_{l, k}\left[N_{\mathrm{RFFT}}\right]\right]$ is obtained. Subsequently, the $N_{\text {CFFT }}$ point FFT operation on $\mathbf{Y}_{l, k}$ in the chirp domain is conducted where $N_{\mathrm{CFFT}}$ is the number of points for the FFT in the chirp domain. This gives the range and velocity map with $N_{\mathrm{RFFT}} \times N_{\mathrm{CFFT}}$. Finally, with an $N_{\text {AFFT-point }}$ FFT operation on the range and velocity map with $N_{\mathrm{RFFT}} \times N_{\mathrm{CFFT}}$ in the array domain, the 3D map which reflects the range, velocity and DOA is obtained. However, in order to perform a 3D FFT, very high complexity is required, as is well known. This causes the processing time and cost to increase.

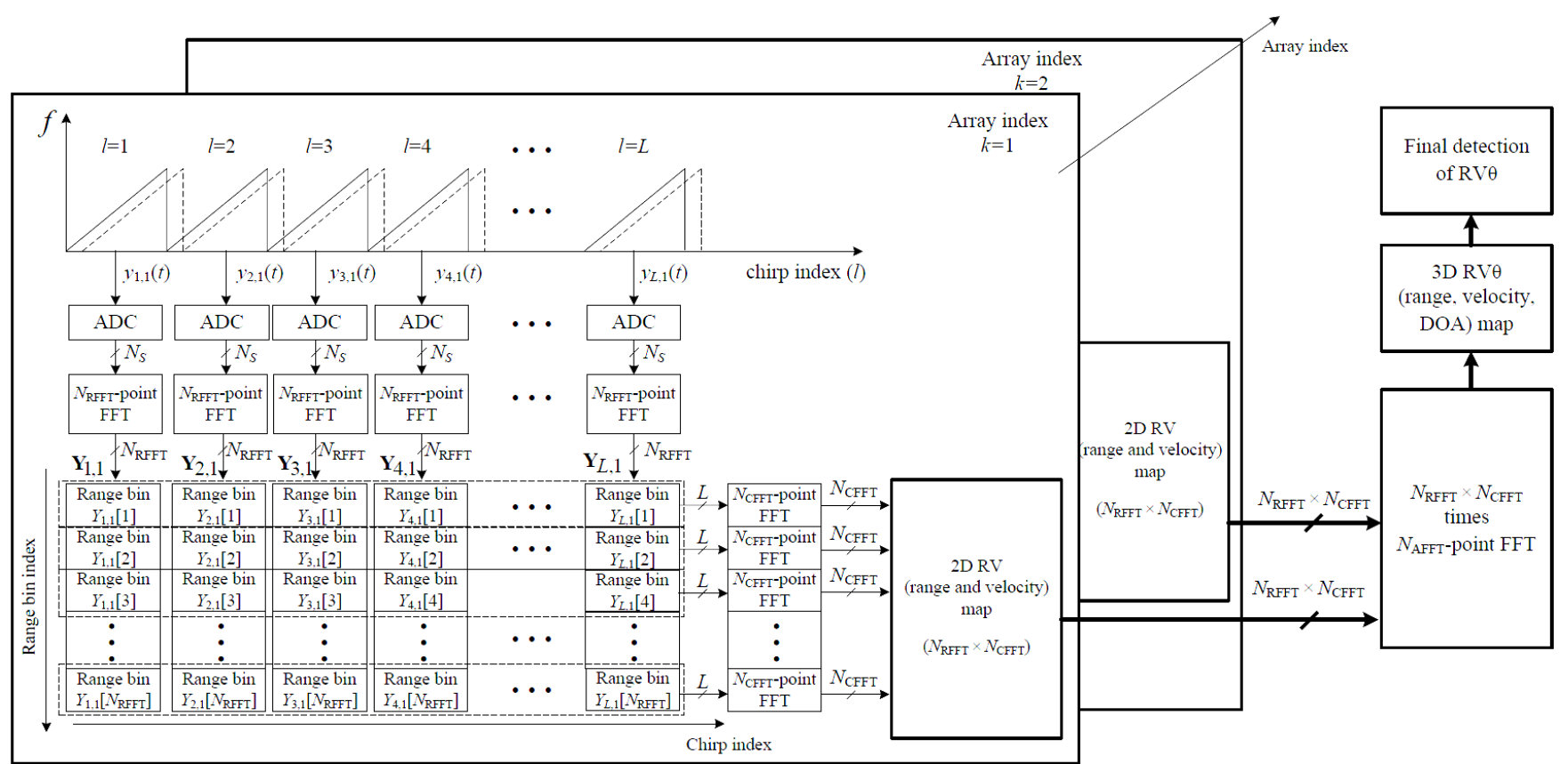

Fig. 3. The conventional 3D FFT FMCW radar detection algorithm.

Meanwhile, in order to reduce the computational complexity of multi-dimensional FFT operations, FFT algorithms with reduced dimensions have been proposed [16]. In these algorithms, the range of the FFT output is initially determined, after which the peak of the magnitude of the FFT output is detected. The authors use the characteristic by which the targets exist only in the range bin with the peak of the magnitude of the FFT output. They conduct a chirp-domain FFT operation on only the range bins in which the target exists. By not performing the chirp-domain FFT on the range bins without a target, they reduce the computational complexity of the multi-dimensional FFT operation. However, these algorithms require additional algorithms such as clutter mitigation or moving target indicator algorithms because they do not distinguish between clutter and the target.

\section{PRoposed FMCW RADAR Algorithm For SURVEILLANCE APPLICATIONS}

In the surveillance radar systems, the first important issue is a quick determination of the presence of the targets of interest. In cases where there are no targets of interest, we do not need to use resources unnecessarily. That is, the detection algorithm should be run only if there is a target of interest. To this end, the proposed method initially determines whether or not a target exists. Parameter detection operations are performed after it is determined that a target exists.

Figure 4 illustrates the structure of the proposed algorithm. As shown in Fig. 4, the proposed algorithm properly selects two beat signals among all $L$ beat signals according to the velocity of interesting target; that is, the $p$-th and the $q$-th beat signals, $y_{p, k}[n]$ and $y_{q, k}[n]$, are selected. We then subtract the two signals, with this action denoted by $d_{p q, k}[n]$, as follows

$$
\begin{gathered}
d_{p q, k}[n]=y_{p, k}[n]-y_{q, k}[n]= \\
=a x_{b}\left[n t_{s}\right] \psi^{k}\left(v^{p}-v^{q}\right)+w_{p, k}[n]-w_{q, k}[n] .
\end{gathered}
$$

Generally, clutter or stationary targets do not move. Accordingly, the Doppler effect does not occur. On the other hand, when the target moves, the Doppler effect arises, and the phase changes as the index of the chirp increases. If there is no moving target, the Doppler frequency equals zero; i.e., $f_{D}=0$. Therefore, only the noise terms remains in (9) because $v^{p}(0)=v^{q}(0)$. This implies that only the noise term is input as the $N_{\text {RFFT-point }}$ FFT input. On the other hand, if there is a moving target, $v^{p}$ and $v^{q}$ are not equal except when $p=q$ and $q=p+i T$, where $i$ is the integer number and $T$ is the chirp duration. Therefore, for simplicity, by omitting the noise terms, (8) is simply expressed as

$$
d_{p q, k}[n]=\alpha x_{b}\left[n t_{s}\right] \psi^{k}
$$

where $\alpha=a\left(v^{p}-v^{q}\right)$. From (10), it can be seen that the beat signal $x_{b}\left[n t_{s}\right]$ including the range information and the DOA term $\psi^{k}$ still exists. 
Here, we properly select two chirps, i.e., $p$ and $q$. This is done because we want to avoid a case in which the proposed algorithm does not work properly. For example, if the phase changes at the two chirps are symmetrical, the output after subtraction may be zero, as if there is no moving target. However, the duration of one frame is relatively short compared to the moving time of the target. By properly selecting two chirps, we can avoid cases in which the phase changes of two chirps are symmetrical.



Fig. 4. The structure of range detection of the proposed algorithm.

Figure 5 shows the waveforms of beat signals and their difference according to a sample index for stationary and moving targets, respectively, when $l=1$ and 3 . As shown in Fig. 5(a), in the case of the stationary target, it can be seen that the difference between the two beat signals becomes almost zero. In the other hand, in the case of the moving target, the difference between the two beat signals follows a sinusoidal waveform with the beat frequency as shown in Fig. 5(b).

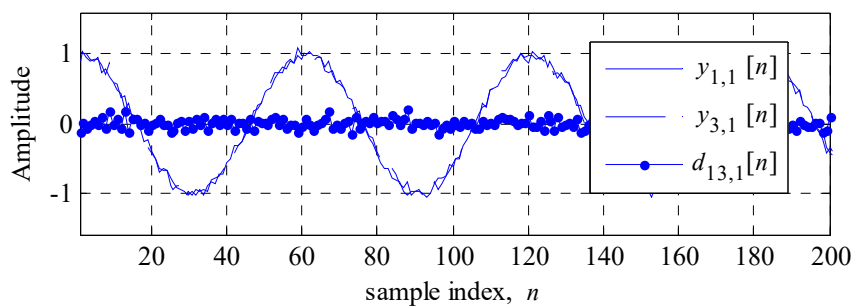

(a)

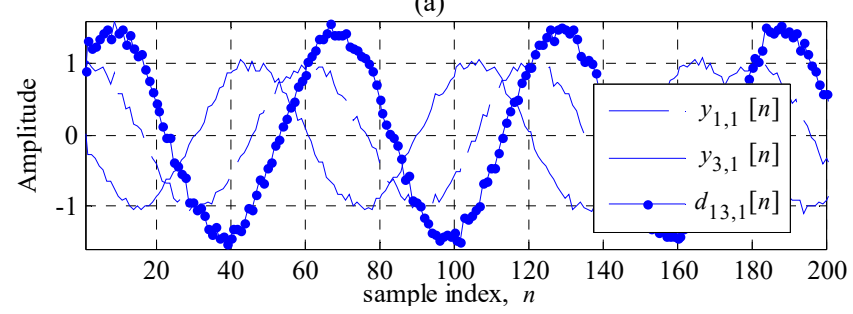

(b)

Fig. 5. Difference between beat signals with $l=1$ and 3 according to sample index in the cases of stationary and moving targets: (a) stationary target; (b) moving target.
In order to estimate the range of the target, we perform FFT on $d_{p q, k}[n]$ with respect to the sample domain $n$, as in (8); thus, the $N_{\text {RFFT }}-$ FFT output of $d_{p q, k}[n]$ denoted by $D_{p q, k}[u]$ is obtained. We then obtain the index $i_{\text {peak }}$ corresponding to the beat frequency of $d_{p q, k}[n]$ through the peak detection of $\left|D_{p q, k}[u]\right|$ for $0 \leq u \leq N_{\mathrm{RFFT}}-1$. Figure 6 shows an example of the result of the peak detection of $\left|D_{p q, k}[u]\right|$ when $i_{\text {peak }}=35$. From the obtained $i_{\text {peak }}$, the estimated range $\hat{R}$ is calculated as [16]

$$
\hat{R}=\frac{c \times \hat{\tau}}{2}=\frac{c \times \hat{f}_{b}}{2 \mu}=\frac{c \times i_{\text {peak }} \times f_{s}}{2 \mu N_{\mathrm{RFFT}}},
$$

where $c$ is the speed of light, $\hat{\tau}$ is the estimated delay, and $\hat{f}_{b}$ is the estimated beat frequency.

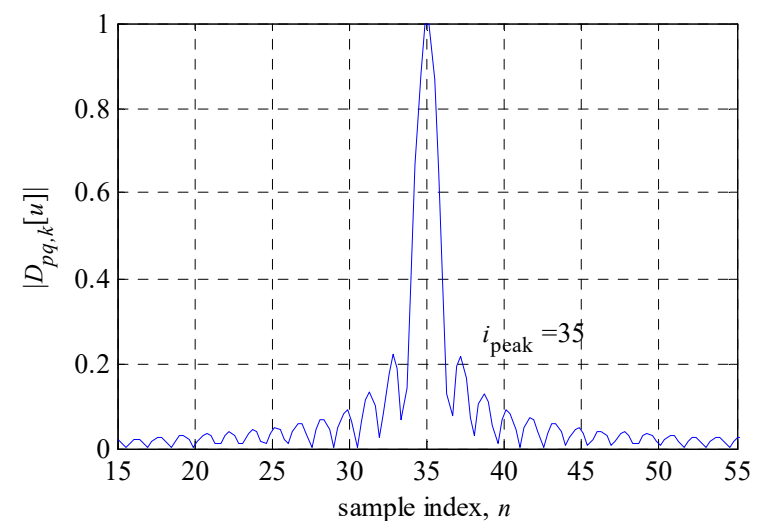

Fig. 6. Example of the result of peak detection of $\left|D_{p q, k}[u]\right|$.

Furthermore, we use only two RX antennas in order to ensure a low cost and low complexity. The proposed algorithm does not perform array-domain FFT in order to detect the DOA information. In the proposed algorithm, the DOA is estimated using the phase change between two arrays instead of an array-domain FFT, as follows [17]

$$
\begin{aligned}
& \hat{\theta}=\sin ^{-1}\left[\angle\left(Y_{p, 1}^{*}\left[i_{\text {peak }}\right] Y_{p, 2}\left[i_{\text {peak }}\right]\right)\right]= \\
& =\sin ^{-1}[\angle(\beta \exp (j 2 \pi d \sin (\theta) / \lambda))],
\end{aligned}
$$

where $\beta$ denotes the term apart from except DOA term in (3). Of course, this simple DOA estimation is only possible in the condition with a single target. However, even with multiple targets, if the ranges or velocities of the targets are not identical, the outcome will be similar to that of a single-target condition in a FMCW radar system [10].

\section{Simulation Results}

This section addresses the simulation results in order to observe the feasibility of the proposed algorithm. The parameters for the simulation of FMCW radar system are shown in Table I. In order to consider the multiple targets condition, the number of targets is set to four and the 4th target is set to stationary. 
TABLE I. PARAMETERS FOR SIMULATION.

\begin{tabular}{|c|c|}
\hline Parameter & Value \\
\hline Center frequency $(\mathrm{GHz})$ & 24 \\
\hline Bandwidth $(\mathrm{MHz})$ & 200 \\
\hline Symbol duration $(\mu \mathrm{s})$ & 80 \\
\hline Sampling rate $(\mathrm{MHz})$ & 5 \\
\hline Number of chirps & 40 \\
\hline Distance of targets $(\mathrm{m})$ & {$[10,15,20,25]$} \\
\hline Speed of targets $(\mathrm{m} / \mathrm{s})$ & {$[10,10,15,0]$} \\
\hline
\end{tabular}

Figure 7 shows the snapshot of the simulation result of range estimation by the proposed scheme. Even though the number of targets is four, there are only three peaks. This is because the $4^{\text {th }}$ target is stationary and thus only three moving targets are detected. This result implies that the proposed scheme distinguishes between stationary and moving targets by using only two chirps.

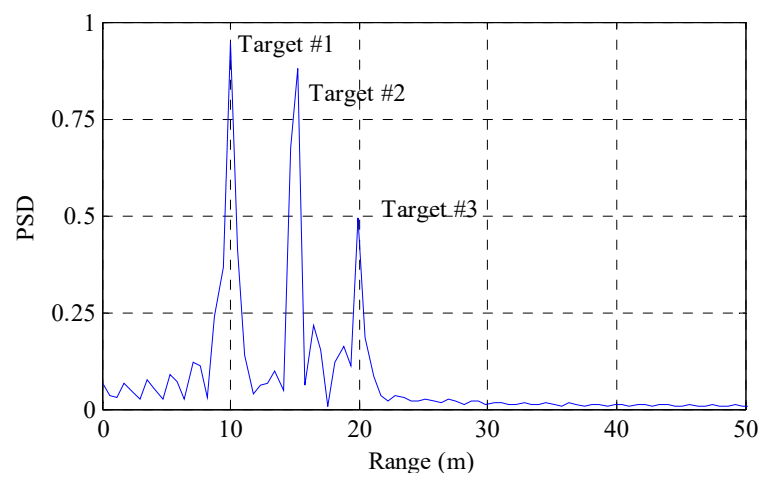

Fig. 7. Snapshot of the result of detection of the proposed scheme.

Figure 8 shows the root mean square error (RMSE) of range estimation of the proposed scheme. In order to observe the effect by complex amplitude of beat signal, the simulation of two cases is performed. Fist, in Case I, the magnitude and phase terms of the complex amplitude $a$ in (7) are set to be uniformly and randomly distributed between 0 and 1 and 0 and $2 \pi$, respectively, i.e., $|a| \in[0,1]$ and $\measuredangle a \in[0,2 \pi]$. On the other hand, in Case II, the magnitude term $|a|$ is fixed to unit amplitude, i.e., $|a|=1$ but the phase term $\measuredangle a$ is set to be uniformly and randomly distributed between $\measuredangle a \in[0,2 \pi]$. In the case of Case I, the performance is degraded in the low SNR region because of fluctuation of magnitude. However, both of two cases achieve the almost same RMSE in the region of $\mathrm{SNR}>10 \mathrm{~dB}$.

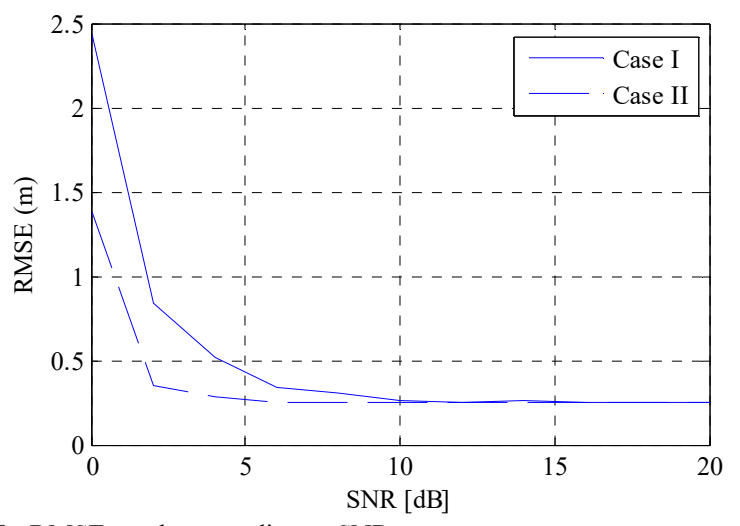

Fig. 8. RMSE results according to SNR.

\section{EXPERIMENT}

In order to confirm the performance of the proposed algorithm using two chirps in an actual environment, we performed a variety of experiments inside an advanced radar technology (ART) laboratory and an anechoic chamber located at DGIST in South Korea [17]. By using the chamber environment, we can find the exact positions of the targets to assess the performance of the algorithm by avoiding undesired echo terms. Then, we show the results of actual human movement by the experiment in the ART laboratory.

\section{A. Front-end and Back-end Modules and Radar Signal Processing}

From Fig. 9 to Fig. 11 show a block diagram of the front-end module (FEM), antenna pattern and photo of back end module (BEM) of our implemented FMCW radar system [18]. The implemented FEM utilizes outputs signals of $200 \mathrm{MHz}$ in the range of $24.05 \mathrm{GHz} 24.25 \mathrm{GHz}$ at $10 \mathrm{dBm}$ of power in the TX part of a FMCW radar system [18]. As shown in Fig. 10, the antenna is composed of one TX and three RX antennas. The TX antenna is composed of five antenna element arrays. The BEM is based on the TMS320 DSP processor with maximum $150 \mathrm{MHz}$ [18].

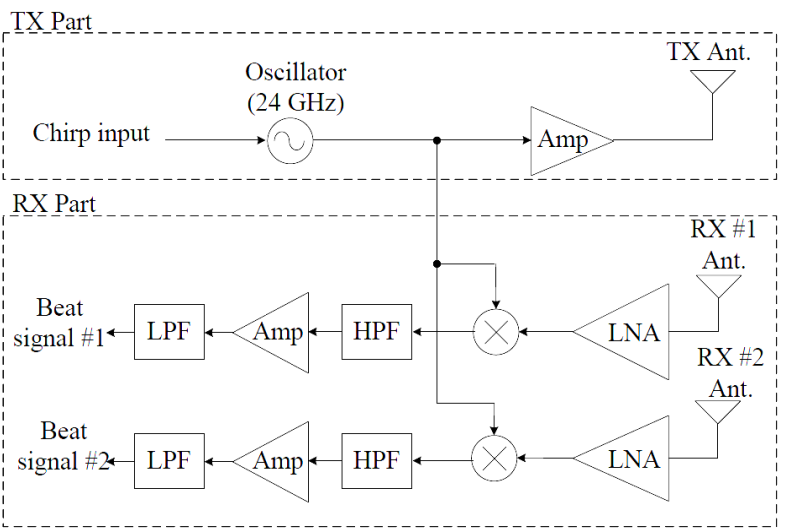

Fig. 9. Functional block of front end modulation (FEM) [18].



Fig. 10. Photo of antenna pattern.

Figure 12 and Fig. 13 show the experiment results of estimated range and DOA of the proposed algorithm which is experimented in the anechoic chamber. For this experiment, out of three targets, two are stationary and only one target is moving. The two stationary targets are located at $3.3 \mathrm{~m}$ and $6.2 \mathrm{~m}$, respectively. The moving target moves between the two stationary targets. As shown in Fig. 12, the power spectral density (PSD) of two stationary targets is insignificant. On the other hand, for the moving target, the 
peak of the PSD is located at approximately $5 \mathrm{~m}$. From this result, the proposed algorithm can detect only the moving target. Fig. 13 shows that the proposed algorithm accurately detects the DOA of moving target.

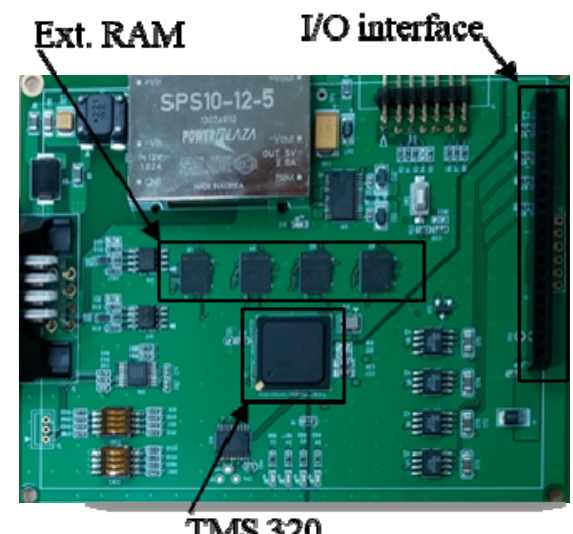

TMS 320

Fig. 11. Photo of back end modulation (BEM) [18].



Fig. 12. Experiment result of range detection of the proposed algorithm.



Fig. 13. Experiment result of DOA detection of the proposed algorithm.

We performed experiments in the ART laboratory when people were actually moving. As shown in Fig. 14(a) person moves from the radar to the wall at a range of $10 \mathrm{~m}$. In order to confirm the performance of the proposed algorithm under several speed conditions, the experiments utilize two speed conditions, i.e., slow mode (walking) and fast mode (jogging).

Figure 15 shows the result of used estimated range according to used frame index $T_{F, i}=i T L$ with the developed surveillance radar system, as performed in Fig. 15. As shown in Fig. 15, the proposed algorithm can detect ranges of several steps, i.e., several speeds condition. In the slow mode, the range of the target changes slowly as the frame index increases. On the other hand, in the fast mode, the change in the range of the target is significant compared to that in the slow mode. These results imply that the proposed algorithm can detect a moving target well in condition which contain clutter and stationary targets despite its low complexity.

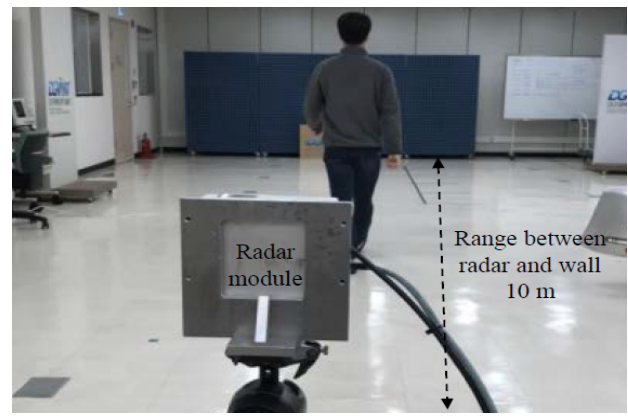

Fig. 14. Photo of surveillance radar experiment for actual moving with single target.

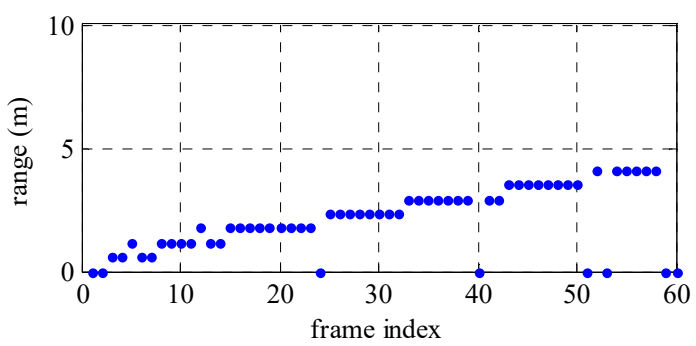

(a)

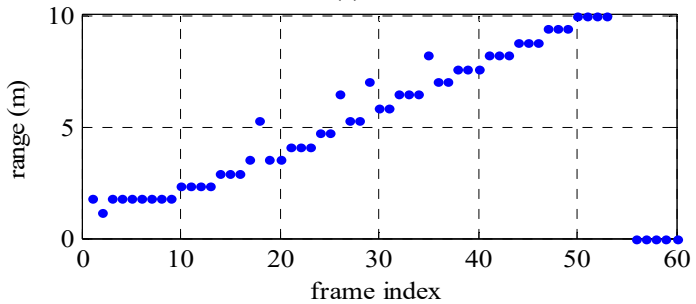

(b)

Fig. 15. Experiment results of range according to frame index (time) by our developed surveillance radar: (a) slow mode; (b) fast mode.

Moreover, in order to verify the detection performance in the multiple targets condition, the experiment is performed in the condition of two persons. As shown in Fig. 16, one person (target 1) walked toward the radar and the other person (target 2) walked away from the radar. Then, target 1 walked away from the radar and target 2 walked toward the radar.

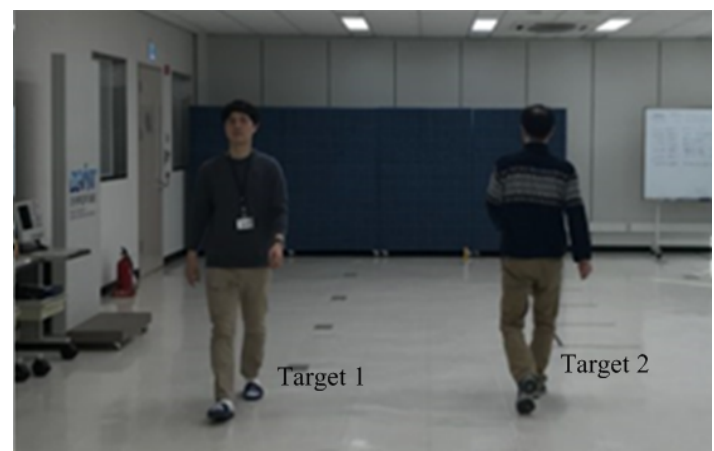

Fig. 16. Photo of surveillance radar experiment for actual moving with multiple targets.

Figure 17 shows the snapshot of detection result (FFT output) of the proposed algorithm. Despite many clutter, the proposed algorithm detects two moving targets.

Figure 18 shows the result of the estimated range according to the frame index for the two persons. In Fig. 18, 
the dashed and solid arrows show the movement of the target 1 and target 2, respectively. Figure 18 shows that according to the elapsed time the movement of two targets is detected well. It implies that the proposed algorithm can detect not only a moving target well but also even in multiple targets under many clutter and stationary obstacle conditions.



Fig. 17. Photo of surveillance radar experiment for actual moving with multiple targets.

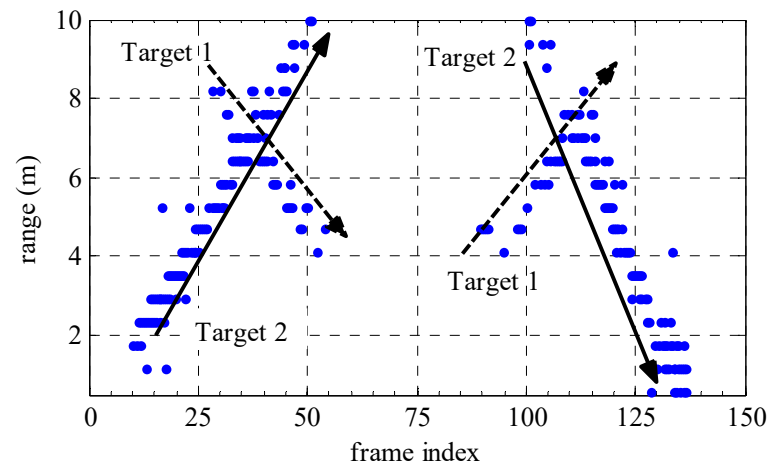

Fig. 18. Experiment results of multiple target detection according to frame index (time).

\section{CONCLUSIONS}

We proposed and implemented $24 \mathrm{GHz}$ FMCW radar algorithm using dual chirps for surveillance applications. In order to reduce the complexity of surveillance FMCW radar algorithm, we performed subtracting between two range-FFT outputs, instead of performing full 2D FFT. In order to verify the effect of the proposed scheme, the simulation results are shown such as RMSE and detection performance in the case of two kinds of amplitudes. Especially, it can be seen that RMSEs of two conditions become almost similar when SNR is larger than $10 \mathrm{~dB}$ region. In addition, experiments were conducted on moving and stationary targets in an anechoic chamber in order to verify the implementation of the proposed scheme. Furthermore, it was practically confirmed that the multiple targets are well detected even in the clutter-vulnerable environment even though it used only 2 chirps among the total 40 chirps.

\section{REFERENCES}

[1] M. Richards, M. A. Richards, J. A. Scheer, W. A. Holm, Principles of Modern Radar: Basic Principles. Institution of Engineering and Technology. 2010. DOI: 10.1049/sbra021e.

[2] J. Li, P. Stoica, "MIMO radar with collocated antennas", IEEE Signal Process. Mag., vol. 24, no. 5, pp. 106-114, 2007. DOI: 10.1109/msp.2007.904812.

[3] H. H. Meinel, "Evolving automotive radar: From the very beginnings into the future", in Proc. European Conf. Antennas and Propagation, Hague, Nethelands, 2014, pp. 3107-3114. DOI: 10.1109/eucap.2014. 6902486.

[4] G. Reina, D. Johnson, J. Underwood, "Radar sensing for intelligent vehicles in urban environments", Sensors, vol. 15, no. 6, pp. 14661-14678, 2015. DOI: 10.3390/s150614661.

[5] A. Natarajan, A. Komijani, X. Guan, A. Babakhani, A. Hajimiri, "A 77-GHz phased-array transceiver with on-chip antennas in silicon: [3] Transmitter and local LO-path phase shifting", IEEE J. Solid-state circuits, vol. 41, no. 12, pp. 2807-2819, 2006. DOI: 10.1109/jssc. 2006.884817.

[6] C. Hu, Y. Liu, H. Meng, "Randomized switched antenna array FMCW radar for automotive applications", IEEE Trans. on Vehi. Tech., vol. 63, no. 8, 2014. DOI: 10.1109/tvt.2014.2308895.

[7] A. Tessmann, S. Kudszus, T. Feltgen, M. Riessle, C. Sklarczyk, W. H. Haydl, "Compact single-chip W-band FMCW radar modules for commercial high-resolution sensor applications", IEEE Trans. on Microwave Theory and Tech., vol. 50, no. 12, pp. 2995-2997, 2002. DOI: $10.1109 /$ tmtt.2002.805162.

[8] D. C. W. Low, K. W. Chang, R. Lin, E. W. Lin, H. Wang, M. Biedenbender, G. S. Dow, B. R. Allen, "A single-chip W-band transceiver with front-end switching receiver for FMCW radar applications", IEEE Microwave Millimeter-Wave Monolithic Circuits Symp. Dig., pp. 225-228, 1995. DOI: 10.1109/mcs.1995.470952.

[9] A. G. Stove, "Linear FMCW radar techniques", in Proc. Inst. Elect. Eng. F-Radar Signal Process., 1992, vol. 139, no. 5, pp. 343-350. DOI: 10.1049/ip-f-2.1992.0048.

[10] E. Hyun, Y. Jin, J. Lee, "Design and implementation of $24 \mathrm{GHz}$ multichannel FMCW surveillance radar with a software-reconfigurable baseband", Journal of Sensors, 2017. DOI: 10.1155/2017/3148237.

[11] S. Saponara, B. Neri, "Radar sensor signal acquisition and multidimensional FFT processing for surveillance applications in transport systems", IEEE Trans. on Instrumentation and Measurement, vol. 66, no. 4, 2017. DOI: 10.1109/tim.2016.2640518.

[12] S. Saponara, B. Neri, "Radar sensor signal acquisition and 3D FFT processing for smart mobility surveillance systems", in Proc. IEEE Sens. Appl. Symp., 2016, pp. 1-6. DOI: 10.1109/sas.2016.7479883.

[13] S. Saponara, S. Lischi, R., Massini, L. Musetti, D. Stagliano, F. Berizzi, B. Neri, "Low cost FMCW radar design and implementation for Harbour surveillance applications", Applications in Electronics Pervading Industry, Environment Society, pp 139-144, 2015. DOI: 10.1007/978-3-319-20227-3 18.

[14] D. Tarchi, G. Guglieri, M. Vespe, C. Gioia, F. Sermi, V. Kyovtorov, "Search and rescue: surveillance support from RPAs radar", in Proc. IEEE Navigation Conf. 2017. DOI: 10.1109/euronav.2017.7954216.

[15] M. Caris, W. Johannes, S. Stanko, N. Pohl, "Millimeter wave radar for perimeter surveillance and detection of MAVs", in Proc. IEEE Radar Symposium, 2015. DOI: 10.1109/irs.2015.7226314.

[16] C. Wang, H. Liu, G. Han, X. Jing, "Automotive FMCW radar-enhanced range estimation via a local resampling Fourier transform", International Journal of Advanced Robotic Systems, vol. 13, 2017. DOI: 10.5772/62179.

[17] S. Kim, D. Oh, J. Lee, "Joint DFT-ESPRIT estimation for TOA and DOA in vehicle FMCW radars", IEEE Antennas and Wireless Propag. Lett., vol. 14, pp. 1710-1713, 2015. DOI: 10.1109/lawp.2015.2420579.

[18] Y. Jin, Y. Ju, J. Lee, "Development of real-time LCA system based on automotive radar", in Proc. (ICONS 2016), 2016. DOI: 10.1109/radar.2014.7060385. 